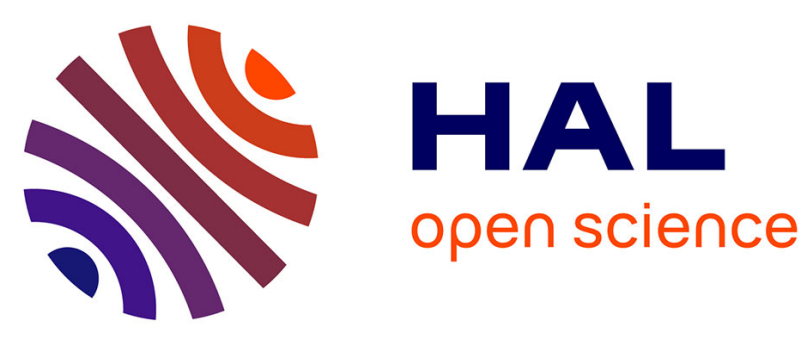

\title{
Reliable MAC design for ambient assisted living: Moving the coordination to the cloud
}

\author{
Elli Kartsakli, A. Antonopoulos, Aris Lalos, Stefano Tennina, Marco Di
} Renzo, Luis Alonso, Christos Verikoukis

\section{- To cite this version:}

Elli Kartsakli, A. Antonopoulos, Aris Lalos, Stefano Tennina, Marco Di Renzo, et al.. Reliable MAC design for ambient assisted living: Moving the coordination to the cloud. IEEE Communications Magazine, 2015, 53 (1), pp.78-86. 10.1109/mcom.2015.7010519 . hal-01270586

\section{HAL Id: hal-01270586}

https://hal-centralesupelec.archives-ouvertes.fr/hal-01270586

Submitted on 9 Jul 2020

HAL is a multi-disciplinary open access archive for the deposit and dissemination of scientific research documents, whether they are published or not. The documents may come from teaching and research institutions in France or abroad, or from public or private research centers.
L'archive ouverte pluridisciplinaire HAL, est destinée au dépôt et à la diffusion de documents scientifiques de niveau recherche, publiés ou non, émanant des établissements d'enseignement et de recherche français ou étrangers, des laboratoires publics ou privés. 


\title{
Reliable MAC design for Ambient Assisted Living: Moving the Coordination to the Cloud
}

\author{
Elli Kartsakli*, Angelos Antonopoulos* ${ }^{* \dagger}$, Aris S. Lalos*, Stefano Tennina ${ }^{\ddagger}$, \\ Marco Di Renzo ${ }^{\S}$, Luis Alonso*, and Christos Verikoukis ${ }^{\dagger}$ \\ *Signal Theory and Communications Dept., Technical University of Catalonia, Barcelona, Spain \\ ${ }^{\dagger}$ Telecommunications Technological Centre of Catalonia (CTTC), Castelldefels, Spain \\ ${ }^{\ddagger}$ WEST Aquila srl, University of L’Aquila, Italy \\ ${ }^{\S}$ Laboratory of Signals and Systems (L2S), Univ Paris-Sud (Paris), France
}

\begin{abstract}
Ambient Assisted Living (AAL) technologies constitute a new paradigm that promises quality of life enhancements in chronic-care patients and elderly people. From a communication perspective, they involve heterogeneous deployments of body and ambient sensors in compex, multihop topologies. Such networks can significantly benefit from the application of cooperative schemes based on network coding, where random linear combinations of the original data packets are transmitted in order to exploit diversity. Nevertheless, network coordination is sometimes required to obtain the full potential of these schemes, especially in the presence of channel errors, requiring the design of efficient, reliable and versatile Medium Access Control (MAC) protocols. Motivated by the recent advances in cloud computing, we investigate the possibility of transferring the network coordination to the cloud while maintaining the data exchange and storage at a local data plane. Hence, we design a general framework for the development of cloud-assisted protocols for AAL applications and propose a high-performance and errorresilient MAC scheme with cloud capabilities.

Index Terms-Pervasive healthcare, Cloud architecture, Ambient Assisted Living, MAC protocol design.
\end{abstract}

\section{INTRODUCTION}

Demographic trends of the last decades reveal an indisputable truth: the world's population is gradually getting older. According to the World Health Organization (WHO) [1], the proportion of the global population aged over 60 years is expected to double from about $11 \%$ in 2013 to $22 \%$ by 2050. One of the contributing factors to population aging is the fact that, thanks to advances in modern medicine and sanitation, life expectancy tends to increase. In fact, the average life span globally is projected to rise from 69 years in 2005-2010 to 76 years in 2045-2050 [1].
This increasing longevity is unprecedented and comes with many challenges for the individual and the society. Cardiovascular and respiratory diseases, cancer, diabetes, disabilities and cognitive impairments are chronic conditions that are more prevalent on older age groups. Significant resources are required for the management of these diseases, including frequent and lengthy hospitalizations, long-term monitoring and complex interactions with medical professionals. As a result, the quality of life of the chronic-care and elderly patients can be severely compromised, increasing the strain on their families and caregivers and placing considerable economic burden on the healthcare system.

The pervasive use of wireless communication technologies can play a fundamental role in improving healthcare delivery and ensuring cost-effective and patient-centered disease management and prevention. Recently, Ambient Assisted Living (AAL) technologies have emerged as a new paradigm that employs ambient intelligent tools [2] to provide a smart and enhanced living environment. AAL systems promote safety and autonomy, offer assistance with daily activities and ensure access to social and medical services, thus supporting independent living and encouraging a healthier lifestyle [3]. Typical AAL environments include smart homes [4] and apartments, geriatric or rehabilitation residences and even hospital wards dedicated to long-term care.

Even though there is no unified framework for the design of AAL systems, some key components of their architecture can be identified. To begin with, unobtrusive health, activity and ambient monitoring lies in the heart of a typical AAL solution. Wearable or implantable sensors are employed to 
measure vital signs (e.g., body temperature, brain activity, heart rate, etc.) and activity patterns (e.g., walking, fall detection, etc.). Ambient sensors are also deployed to obtain context information on the physical environment (e.g., temperature, lighting, etc.) and the user location. Apart from sensors, AAL systems may include acting devices, such as medical actuators (e.g., insulin pumps), robotic devices and domotic systems for home automation. All these components are glued together through middleware platforms [5] that integrate software algorithms and reasoning engines for processing and analyzing the collected data [6], provide user-friendly interfaces [7] and facilitate the development of AAL application services.

From a communication perspective, an AAL system usually requires the joint deployment of mobile Wireless Body Area Networks (WBANs), formed by the sensors deployed in the vicinity of or within the body of moving users, and static ambient Wireless Sensor Networks (WSNs). In this heterogeneous scenario, the low-power short-range wireless technologies specified within the standards IEEE 802.15.6 and IEEE 802.15.4 for WBANs and WSNs, respectively, are usually employed for the sensor interconnection [8]. Although these standards define the basic mechanisms for channel access and transmissions, there are still many open challenges in order to ensure energy-efficient and reliable communication.

Given the dense deployment of multiple sensors within a specific area, it is possible to achieve significant enhancements by exploiting diversity through node cooperation. The key idea of cooperative schemes is to encourage nodes to act as relays and forward information overheard by neighboring transmissions. Furthermore, with the introduction of network coding, the intermediate nodes can combine and process different received information flows, in order to achieve performance improvements even in resource constrained networks such as WBANs [9]. However, the benefits of network coding cannot be fully exploited in the presence of channel errors, and under hostile indoor and body area propagation environments the amount of redundancy required to ensure successful decoding can be prohibiting.

An effective way to drastically reduce redundant retransmissions without compromising performance is by enabling the exchange of information among the relays, allowing them to act in a coordinated manner. Hence, novel Medium Access Control (MAC) protocols must be envisioned to handle data transmissions and relay cooperation in such dynamic and multihop topologies. Nevertheless, as deployments tend to grow in complexity, the network coordination becomes overwhelming due to the high number of nodes and the large amount of collected data. Furthermore, distributed approaches may require the nodes to have increasing processing power and storage capabilities.

Recent advances in cloud computing offer an alternative solution to mitigate these challenges. This new paradigm, which is revolutionizing the way information systems are designed, is based on a shared pool of hardware and software resources that are easily accessible via the Internet and are often managed by third-party providers. Current research on cloud-based pervasive healthcare is still in its initial steps [10], but it seems that there are tremendous opportunities to be seized through the seamless integration of cloud technology and WBANs [11]. Typical cloud services include data storage, processing and hosting of medical application services and interfaces [12], [13].

A less investigated but equally intriguing topic is to employ cloud resources at lower communication layers, to design innovative MAC protocols and routing schemes with increased flexibility and reconfigurability. In one of the few existing works in this context [14], a cloud-assisted MAC protocol has been proposed and implemented for a Wireless Local Area Network (WLAN) deployment. The main idea is to transform the access points into a unified user interface and concentrate MAC layer functions to virtual machines provided by cloud services. This novel concept is rather focused on implementation issues, thus offering a significant practical contribution. Another interesting work in [15] gives some preliminary insights on the use of network coding in complex large-scale networks where multiple relay nodes interacting with each other form a wireless cloud. The presented results show the potential gains of different transmission strategies in a very simple topology. The authors also indicate the need for some level of inter-cloud cooperation in order to ensure the best strategy selection by the relays, which can be a challenging task in large-scale networks. Summarizing, these works show that cloud-based solutions for the MAC 
layer can yield promising results, however there is a need to establish solid frameworks for the implementation of these schemes.

In this article, we present a novel cloud-based architecture conceived for AAL environments where medical and ambient sensors are densely deployed. We envision a scenario where part of the sensor infrastructure is connected to the cloud, forming a network of cloud-enabled relays that can significantly enhance the flow of information within the system. An innovative feature of our framework is that we transfer the network coordination to a central controller, which is located at the cloud and communicates with the nodes through a cloudassisted control plane, whereas we maintain the exchange and storage of data packets to a local data plane.

The remaining of this article is organized as follows. Initially, we describe the proposed cloudassisted framework, defining the different operation planes and the key architecture components. We, then, present a specific case study on the application of network coding in cooperative relay networks, to illustrate the significant potential of the proposed framework. After identifying a major performance weakness of network coding schemes in the presence of link failures, we propose a novel cloud-assisted MAC scheme that employs cloud resources to achieve relay coordination and demonstrate, through simulation-based performance evaluation, the significant performance gains that can be obtained. Then, we provide some suggestions for future lines of research, stemming from the application of the proposed framework to a wide range of applications for AAL environments. Finally, we close the article with some general conclusions.

\section{A Cloud-BASEd Framework For AAL}

The proposed technology agnostic cloud-based framework defines two planes of operation, as shown in Figure 1:

- the cloud-assisted control plane, responsible for the network control and coordination. Control signaling has the form of very short messages exchanged between the nodes and a coordinating entity, which will be denoted as cloud controller, located at the cloud. Control communication takes place through a dedicated high-speed link, employing Ethernet or cellular 3G/4G technologies.

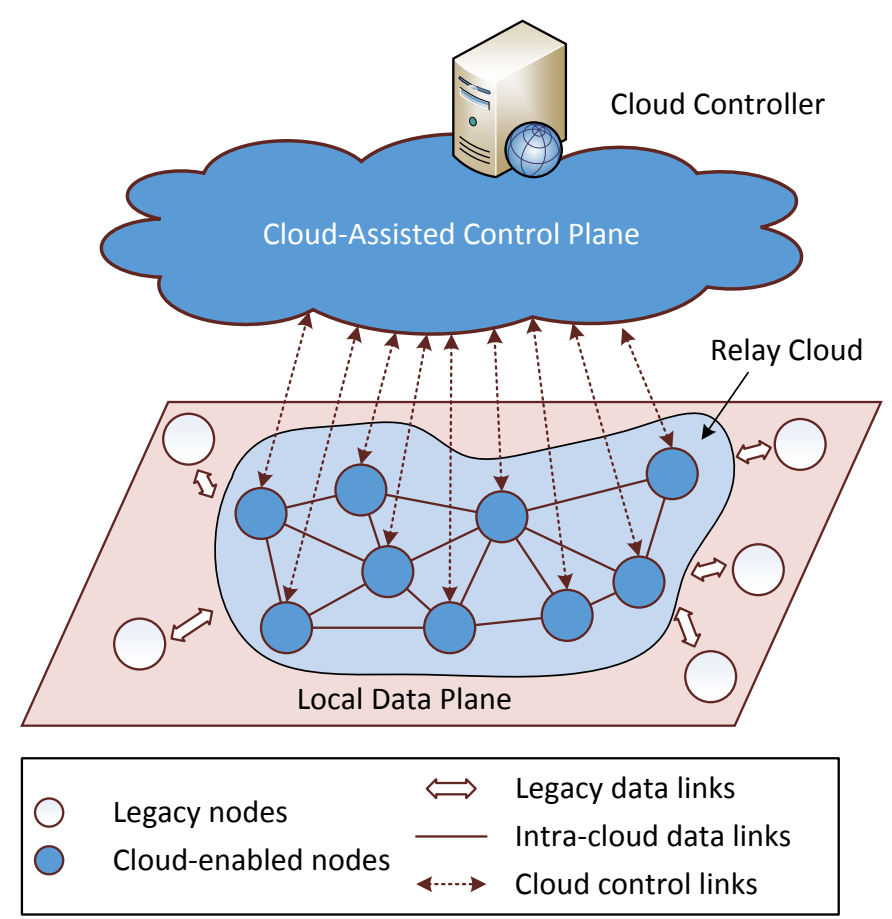

Fig. 1: The proposed cloud-based framework

- the local data plane, responsible for data transmissions among the sensors. To this end, short range technologies are employed, such as the IEEE 802.15.6 standard for WBANs or the IEEE 802.15.4 standard for WSNs. Without going yet into details about the network topology and operation, we assume that multihop communication is supported among the sensors in order to achieve a given goal (e.g., data dissemination or data collection by sink nodes). In addition, different protocols can be considered for the MAC layer, including cooperative policies and network coding schemes.

With this system architecture in mind, the network components can be classified into three categories, according to their operation:

- the cloud controller, which is a coordinating entity located at the cloud, operating only at the control plane. The cloud controller has global knowledge of the network formed by the cloudenabled nodes and can make optimal decisions for the network operation by processing this information.

- the cloud-enabled nodes, which are sensor nodes with enhanced capabilities that can operate in both data and control plane. These nodes play a key role in the proposed framework. 
By being able to exchange control information with the cloud controller, they can act in a coordinated manner, forming a cloud of nodes that enables the implementation of advanced cooperative MAC and routing schemes, thus facilitating significantly the flow of information within the data plane.

- the legacy nodes, which are sensor nodes that can only operate in the data plane. These nodes have more stringent power and processing constraints and can benefit from the enhanced capabilities of the cloud-enabled nodes.

Based on the proposed architecture, the legacy nodes view the network of cloud-enabled relays as a single entity with a unified interface, without the need to be concerned about its structure or individual elements. In addition, crucial global information on the network can be collected by the cloud controller via the control plane. These design features offer several advantages that enable the design of efficient MAC schemes, especially when some level of centralized control is desired.

In the next section, we illustrate one potential application of the proposed framework in AAL environments, by presenting a cloud-assisted MAC scheme that exploits network coding in the presence of channel errors.

\section{Case Study: A Cloud-Assisted MAC PROTOCOL FOR ENHANCED NETWORK CODING RELIABILITY}

Recently, the application of practical random linear network coding (RLNC) schemes, based on the generation and transmission of random linear combinations of the original data packets, is showing promising results, offering enhancements in throughput, reliability and energy efficiency [9]. However, link failures introduced mainly by channel errors can have a detrimental effect on the performance of such schemes. As a solution to this problem, we show how the proposed framework can be applied to the considered scenario and propose CRNC-MAC, an enhanced cloud-assisted MAC protocol that exploits the centralized control capabilities provided by the cloud in order to extract the full potential of RLNC.

\section{A. Problem Statement: The need for coordination} in RLNC-based schemes

In this case study, we consider an AAL facility where ambient sensors are immersed in the environment and residents are monitored through on-body or implanted medical sensors, forming WBANs. Focusing on a given WBAN, we consider that all the sensed data are gathered by the WBAN coordinator and must, in turn, be forwarded to a locally deployed sink node (e.g., a central medical unit). We also assume that no direct communication is possible between a given source (i.e., the WBAN coordinator) and the final destination, hence the use of relay nodes is required. In this scenario, we assume that the role of relays is played by the network of ambient sensors that generally has a mesh multihop topology.

We focus on a typical RLNC scheme in which the source generates $N$ random linear combinations of the $N$ uncoded (original) data packets, by multiplying each packet with a random coefficient drawn by a finite Galois field. Each relay creates new linear combinations of the received packets and forwards them to the destination (or to the next cluster of relays, in the case of multihop topologies). Finally, the destination is able to perform decoding with success and retrieve the original packets, only after receiving at least $N$ independent linear combinations.

This baseline scheme has an inherent weakness in the presence of channel errors that will be illustrated with the help of the example depicted in Figure 2(a). This example focuses on the $m$ th hop of a generic mesh relay network, i.e., on the transmissions that take place between the $(m-1)$ th and the $m$ th relay clusters. Consider that the original source information is contained within four linearly independent packets (A-D) that have reached the $(m-1)$ th relay cluster. Due to random errors introduced by the channel, each relay has received only a subset of these four packets, which are, then, encoded into new RLNC combinations and sent to the next hop. However, assume that, in this example, the only combination that contains packet $\mathrm{D}$ is transmitted by relay $R_{4}$ and is not received by any of the relays of the $m$ th cluster. As a result, all the packets generated at the next hop will be linear combinations of packets A-C, whereas packet $\mathrm{D}$ will be absent from all transmissions beyond this point. Ultimately, the missing degree of freedom 
will impede the decoding process at the destination, causing significant performance degradation.

Given that acknowledgment frames are not employed in multicast transmissions, the most common solution to this problem is the transmission of redundant data copies by the source and the relays. However, in networks with dynamic topology and no previous knowledge of the channel conditions, the calculation of the optimum number of redundant retransmissions is not a trivial task. Another approach is to exploit cooperative diversity by introducing a high number of relays. Even though this may be possible in AAL scenarios with dense sensor deployments, involving too many intermediate nodes in each transmission will lead to new problems, such as increased interference and energy consumption.

\section{B. CRNC-MAC: Introducing relay coordination through the cloud}

In order to tackle the aforementioned problem in a decisive way, it is necessary to introduce some level of coordination among the relays. To this end, we propose CRNC-MAC, a novel cooperative RLNC-based protocol with enhanced robustness against errors, built within the proposed cloudassisted framework. Figure 2(b) gives a high-level description of the proposed MAC protocol. Going back to the proposed framework architecture, we assume, without loss of generality, that the source and the destination are legacy nodes, whereas the ambient sensors are cloud-enabled relays. All data transmissions take place at the local data plane, whereas the cloud-assisted control plane is employed for the exchange of control information between the relays and the cloud controller.

The key concept behind CRNC-MAC is to employ cloud resources for the relay coordination, to ensure that all crucial information from the source is propagated without losses through the multiple hops, thus enabling the successful decoding at the destination. In particular, the cloud controller has the task of verifying the reception of all required information at a hop by hop basis, requesting retransmissions whenever necessary. In the example of Figure 2(b), once the $m$ th hop transmissions are completed, the relays of the $m$ th cluster forward a list of the received packets to the cloud controller via the control plane. If any crucial information is missing, the relays are notified and request a retransmission from the previous cluster.

In continuation, a more detailed description of the protocol operation is given with the help of an example, shown in Figure 3, where the key steps of the algorithm are numbered and indicated within circles. For the sake of simplicity, we consider a two-hop network in which $N=4$ original packets are transmitted by the source through the cluster of $R=3$ cloud-enabled relays.

The protocol operation in each hop is divided into two phases, the dissemination phase and the cloudassisted coordination phase. In the transmission phase of the first hop (step 1 in Figure 3), the source transmits 4 RLNC combinations (packets $P_{1^{-}}$ $P_{4}$ ) of the original data. The cloud-assisted coordination phase has a variable duration and involves communication in both data and control planes. In the beginning of this phase, each relay informs the controller about the packets that have been correctly received, by transmitting a short control message with the sequence numbers of the respective packets (step 2). Depending on whether all the transmitted packets have been received by the relay cloud, the controller will indicate either the need for retransmissions by the source or the successful termination of the dissemination phase. In our example, packet $P_{4}$ has not been received by any of the relays due to channel errors, thus hindering the decoding process at the destination. Hence, through the control plane (step 3), the controller assigns to one of the relays the task of transmitting a request for retransmission (RRT) at the source (step 4). It should be noted that, since the source employs RLNC, it does not need to retransmit the exact missing packets, but only a sufficient number of linearly independent combinations of the original data. In our example, the source transmits one new RLNC packet $P_{5}$ (step 5).

Given that retransmissions are also affected by channel errors, the coordination phase is repeated again, until all packets are correctly received by the relay cluster. In the considered example, $P_{5}$ is received by two of the relays, hence the dissemination phase is completed with success. The controller receives an updated packet list (step 6) and issues a transmission schedule for the next hop, dictating the order and the number of packets to be transmitted by each relay (step 7).

In the dissemination phase of the second hop 


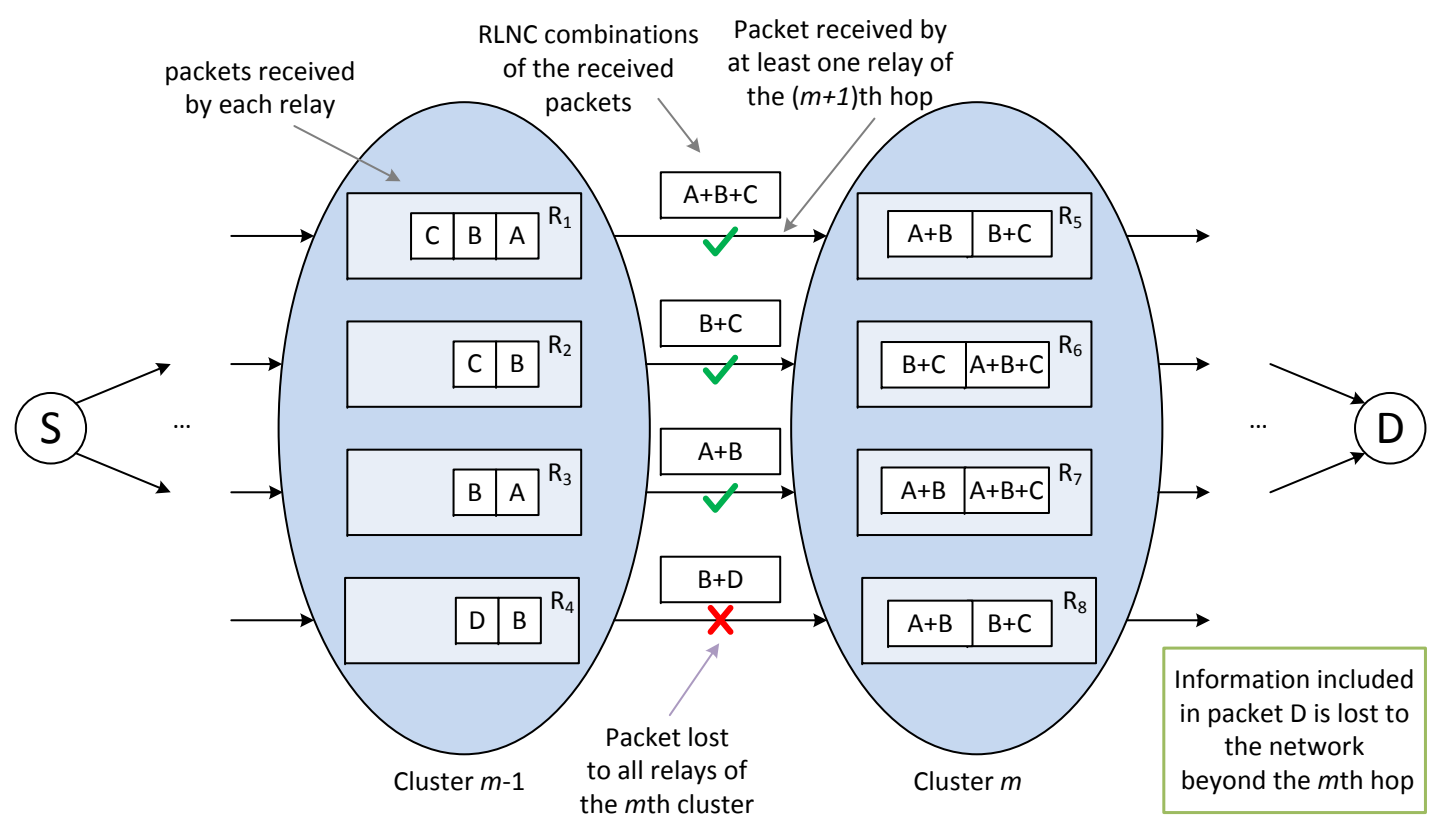

(a) Performance weakness of baseline RLNC schemes under link failures

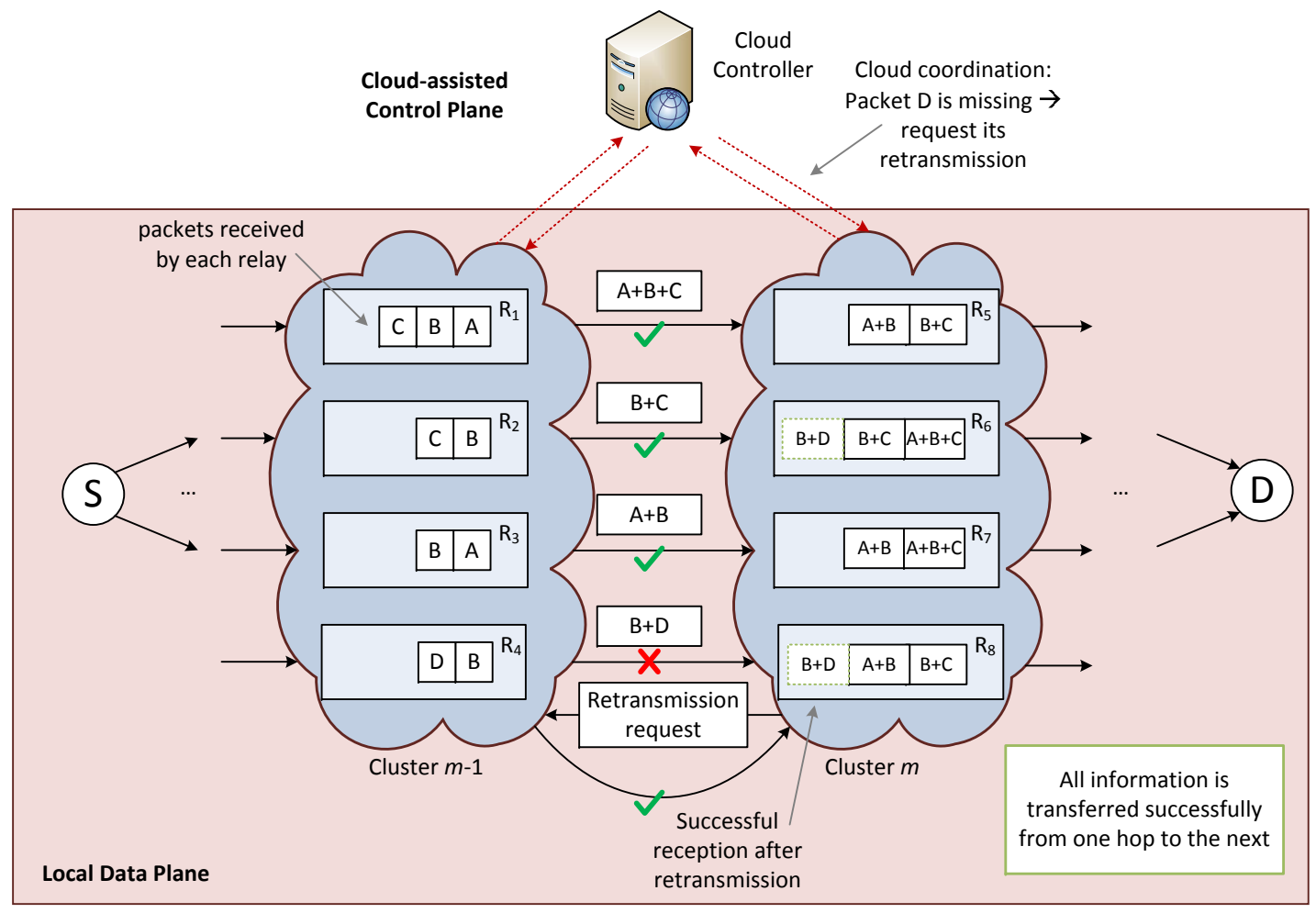

(b) CRNC-MAC provides relay coordination through the cloud-assisted framework

Fig. 2: Problem statement and the proposed cloud-assisted MAC solution 
(step 8), the relays forward RLNC combinations of their received packets $\left(P_{1}^{\prime}-P_{4}^{\prime}\right)$ according to the schedule. In this case, the destination is a legacy node, hence there is no cloud-assisted coordination phase. Hence, transmissions take place until the destination receives a sufficient number of copies to decode the original packets and terminates the relaying phase by transmitting a block acknowledgment (BACK).

\section{Performance Evaluation}

In this section, we show the benefits of cloudassisted coordination through a simulation-based performance evaluation of CRNC-MAC. We have considered a two-hop relay network in order to gain a more intuitive understanding of the existing performance problems and the potential enhancements of the proposed solution. The two-hop topology also gives us a lower bound of the achievable gain, since as the number of hops increases, the need for cloud-assisted coordination becomes more imperative. The erasure channel is modeled as a Bernoulli process with probability of link failure $p$. In terms of simplicity, we assume that the relay links are independent, but have similar average channel conditions in both hops, from source to the relays and from the relays to the destination. Without loss of generality, the narrowband PHY and MAC parameters have been chosen in accordance to the IEEE 802.15.6 standard for WBANs for the data plane communication. The key simulation parameters are summarized in Table I.

With respect to the control plane, we assume error-free and high-speed communication through Ethernet or cellular $3 \mathrm{G} / 4 \mathrm{G}$ technologies. In order to quantify the control overhead, we define $T_{c}$ as the time required for one message exchange between each cloud-enabled relay and the controller. We consider different values for the latency $T_{c}$, ranging from zero, for ideal instantaneous control links, up to $10 \mathrm{~ms}$, which is a realistic values for round-trip delays of very short messages.

We compare the performance of CRNC-MAC with a baseline RLNC-based scheme, denoted as RLNC-MAC, that does not support relay coordination. In RLNC-MAC, the relays transmit their encoded data following the IEEE 802.15.6 contentionbased access rules. Transmissions are either terminated with the reception of a BACK frame after a successful decoding, or when the retransmission limit is reached (set to 10 per relay).

The first set of plots in Figure 4 shows the throughput and energy efficiency performance of CRNC-MAC and RLNC-MAC, with respect to the packet error probability $p$, for $R=3$ relays . Under error-free conditions (i.e., $p=0$ ), CRNC-MAC with no latency $\left(T_{c}=0\right)$ achieves an $18 \%$ throughput improvement with respect to RLNC-MAC, due to the efficient relay transmissions, without collisions and backoff times. However, the key performance gains of CRNC-MAC are appreciated as the error probability grows. In particular, as the channel becomes more hostile, the throughput of RLNC-MAC experiences a steep drop and becomes almost zero for $p=0.6$. This occurs because even though a percentage of the transmitted packets reaches the destination, there are not enough independent linear combinations to enable successful decoding. On the contrary, CRNC-MAC experiences only a slight drop in performance for high error probabilities, due to the increased number of required retransmissions. Nevertheless, successful decoding is always guaranteed, thus yielding an impressive performance gain. Similar results are obtained with respect to the energy efficiency, with the achieved gains ranging from $26 \%$ when no channel errors are considered, to more than $3000 \%$ for $p=0.6$.

When latency is introduced to the cloud communication, the CRNC-MAC throughput is slightly affected, as shown in Figure 4(a). In particular, with respect to the ideal case when $T_{c}=0$, an average throughput degradation of approximately $10 \%$ is observed when $T_{c}=5 \mathrm{~ms}$, increasing to $20 \%$ for $T_{c}=10 \mathrm{~ms}$. Hence, a performance tradeoff is present, depending on the channel quality and the cloud communication latency. Under very good channel conditions (i.e., with $p<0.2$ ), employing cloud resources may not yield significant enhancements, since, for high latencies the obtained throughput is practically the same with the baseline RLNC-MAC. Nevertheless, for medium and high error probabilities, the advantages of the cloudassisted scheme become evident.

The throughput and energy efficiency metrics are also plotted in Figure 5 as a function of the number of relays $R$, for a channel error probability of $p=0.3$. In this case, CRNC-MAC always outperforms the baseline scheme, regardless of the introduced latency. A very interesting observation is 


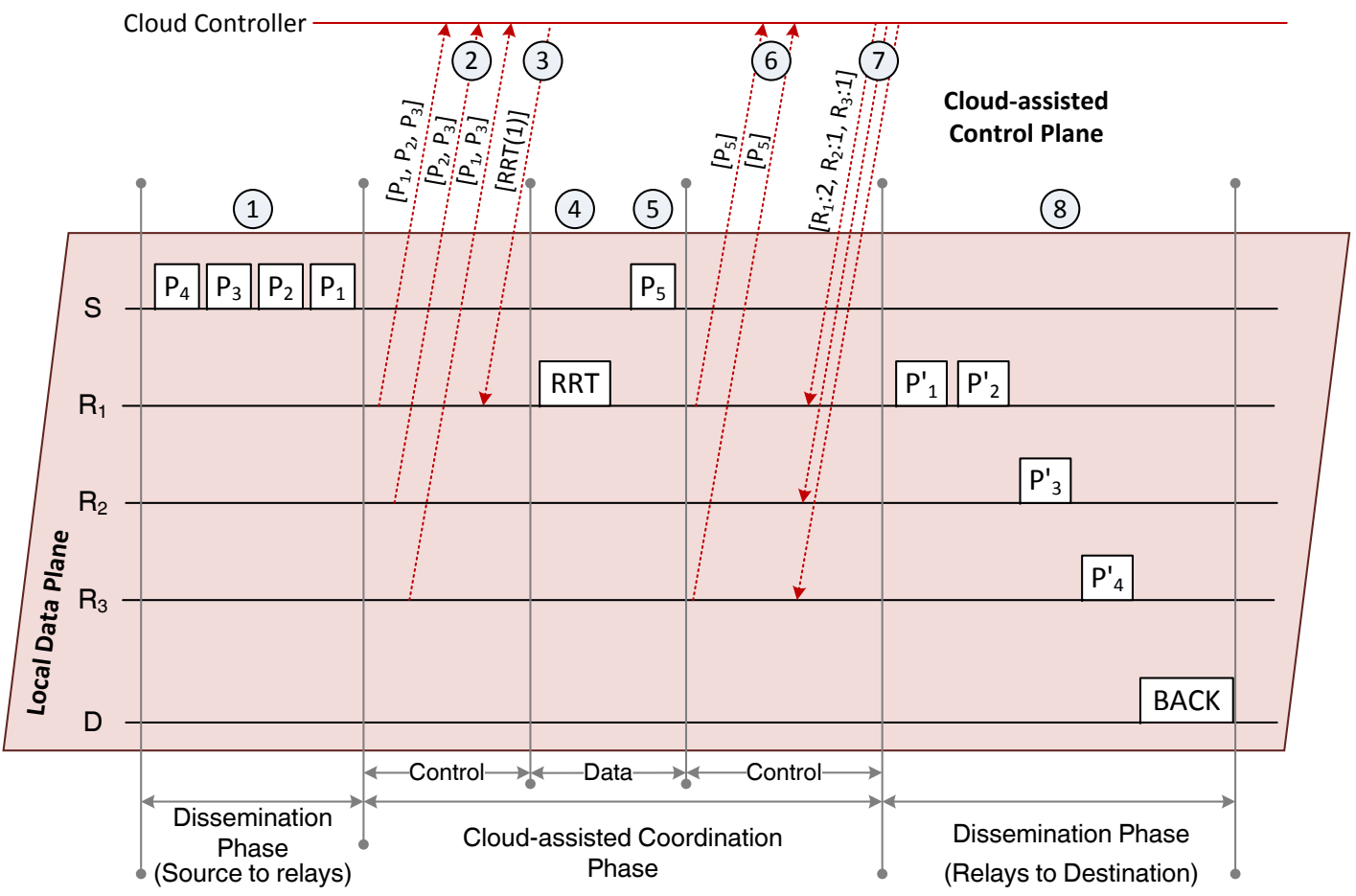

Fig. 3: Example of the CRNC-MAC Operation

TABLE I: Simulation Parameters

\begin{tabular}{llll}
\hline Parameters & Values & Parameters & Values \\
\hline$N$ (packets) & 10 & RTT, BACK (bytes) & 9 \\
$L$ (bytes) & 100 & Data Rate $(\mathrm{kbps})$ & 485.7 \\
Galois Field & $2^{8}$ & Control Rate $(\mathrm{kbps})$ & 121.4 \\
PHY Preamble (bits) & 90 & Transmit power $(\mathrm{mW})$ & 4.6 \\
PHY Header (bits) & 31 & Receive power $(\mathrm{mW})$ & 3.8 \\
MAC Header \& FCS (bytes) & 9 & Idle power $(\mathrm{mW})$ & 0.712 \\
{$\left[C W_{\min }, C W_{\max }\right]$} & {$[16,64]$} & Sleep power $(\mu \mathrm{W})$ & 4 \\
\hline
\end{tabular}

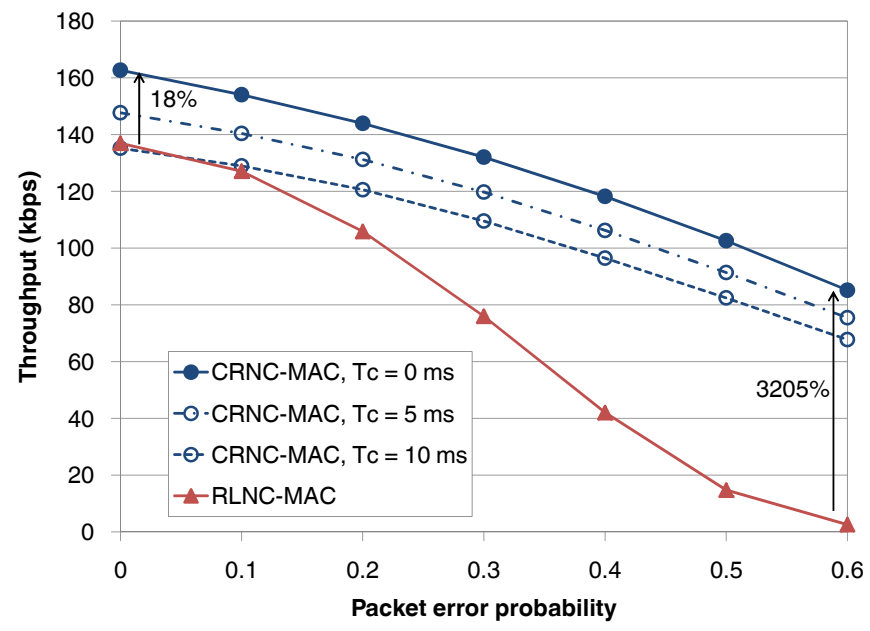

(a) Throughput

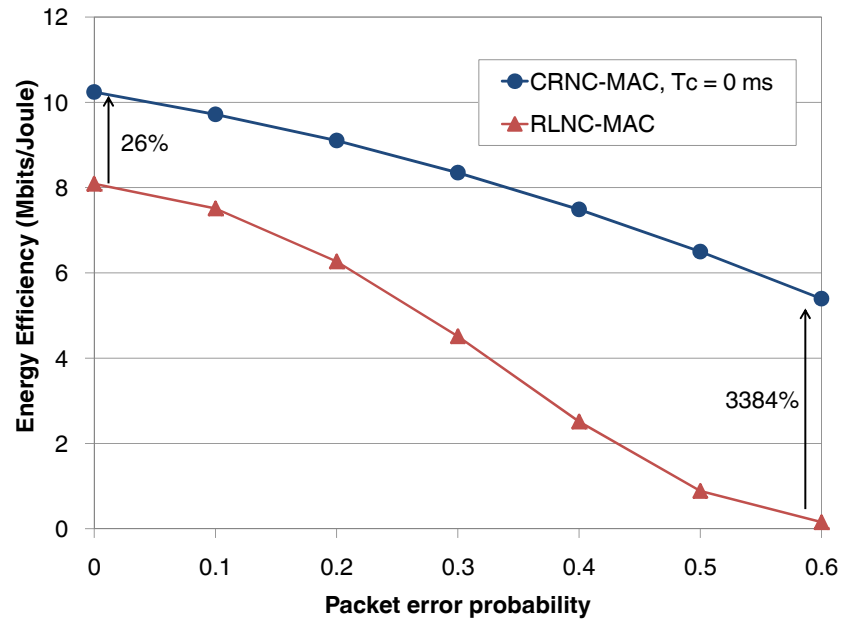

(b) Energy Efficiency

Fig. 4: Performance evaluation with respect to the packet error probability $p$, for $R=3$ relays 
that the throughput performance of CRNC-MAC is not affected much by the number of relays, since the cloud-assisted cooperation enables the achievement of the same diversity gains by a smaller number of relays. As a result, high performance can be achieved even when few relays are available, leading to more energy-efficient communication.

\section{Potential Applications of the ClOUd-ASSISTED FRAMEWORK}

In the previous sections, we have shown how the application of the proposed cloud-assisted framework can enable the design of an efficient MAC scheme that overcomes the performance limitations of RLNC in the presence of channel errors. However, the presented case study is only one example of the benefits that can be obtained through cloudassisted network coordination. In this section, we identify some potential applications of the proposed framework that can serve as the starting point for further research in the design of enhanced protocols for AAL environments.

- Advanced design for MAC and network layer schemes, enabled by the cloud-assisted centralized coordination and the exchange of control information. Besides the presented case study focused on the application of RLNC schemes, different performance goals can be targeted. Accordingly, the acquired feedback can cover different aspects of the network, such as channel quality, buffer state and content, battery level, node temperature, etc., opening a vast number of possibilities for the design of enhanced schemes, including channel and energyaware opportunistic scheduling, collision-free channel access, relay selection schemes, etc. Some of these schemes can be applied to a wide range of scenarios, whereas others may be specifically designed for healthcare applications. An example of the latter case is temperature-based routing [11], that takes into account the effect of heat dissipation by body nodes in order to minimize tissue heating. This scheme requires efficient clustering that can be achieved through cloud-assisted coordination.

- Flexibility in network deployment and maintenance, given that the internal structure of the relay network is invisible to the legacy nodes. This facilitates the deployment of new cloud- enabled nodes or the performance of maintenance tasks, which may often occur in dynamic AAL environments, and increases robustness against device failures. It should also be noted that there is no need for complex neighbor discovery algorithms to detect any changes in the topology, since this information can be readily provided by the cloud controller.

- Mobility and tracking support of legacy nodes in scenarios where the position of the cloudenabled nodes is known (e.g., fixed deployment or GPS (Global Positioning System) capabilities of the relays). A moving legacy node is likely to interact with different cloud-enabled nodes, thus conveying information about its location. Hence, the cloud-enabled nodes play the role of anchor points. By concentrating all this information at the cloud coordinator, it is possible to recreate the trajectory of the mobile node through localization and tracking algorithms. Such applications often form part of AAL systems for the monitoring of patients moving within a constrained environment.

- Distributed storage applications, a recent paradigm based on the concept of storing data with redundancy within the network instead of concentrating them to specific sink nodes. Distributed storage is gaining ground as a means increase reliability in networks with high data contents (e.g., medical records), but requires mechanisms for the efficient reconstruction of the original data. The proposed framework can easily support distributed storage applications, where data generated by the legacy nodes are distributed and stored within the relay cloud. In addition, the central coordination can significantly facilitate the data recovery process, since the cloud controller can obtain feedback on the information stored in each cloud-enabled node.

- Security, which is an indispensable requirement of healthcare applications, due to the sensitive and confidential nature of medical data. The advantage of the proposed framework is that, by defining two planes of operation for data and control, it can support a wide range of security solutions, either centralized or distributed. Let us consider, for example, two main approaches for key management storage in cloud-enabled WBANs, given in [11], and indicate how their weaknesses are mitigated 


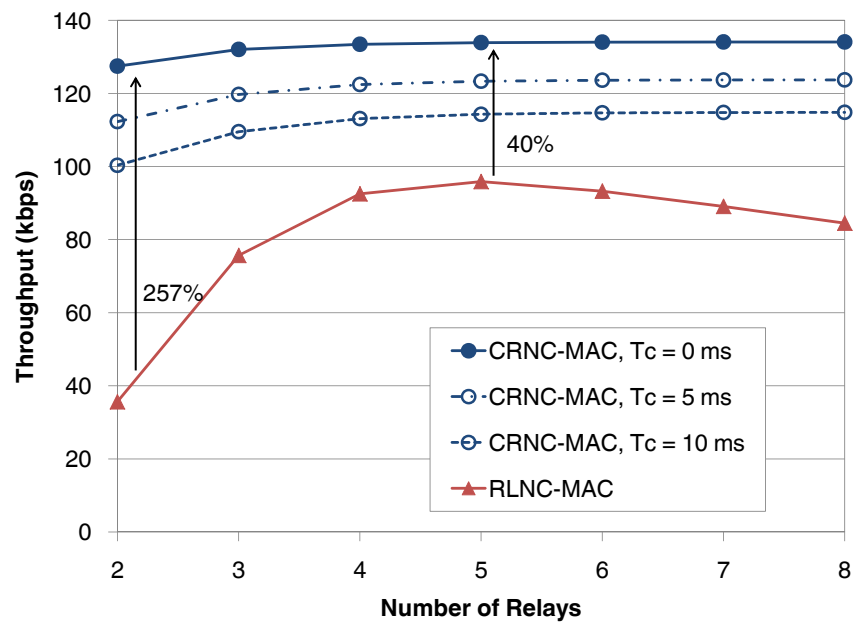

(a) Throughput

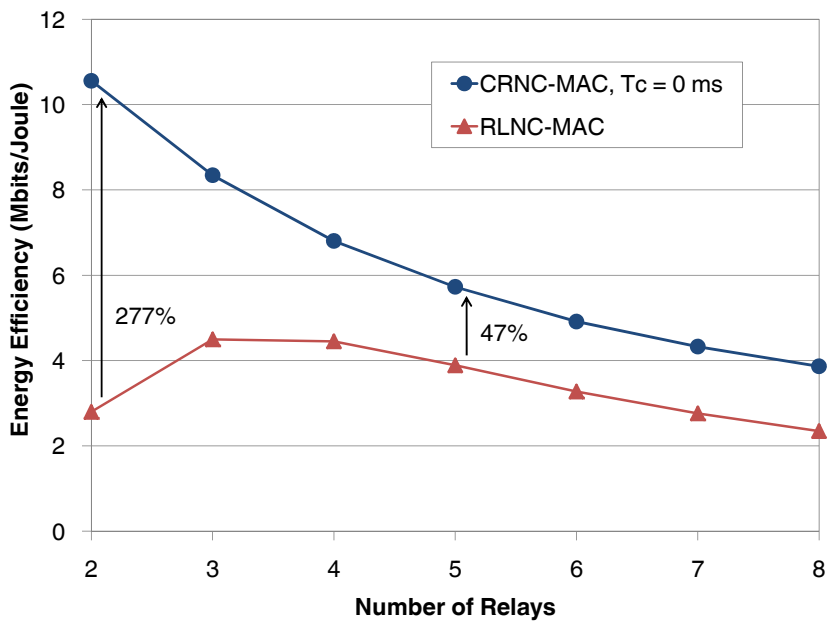

(b) Energy Efficiency

Fig. 5: Performance evaluation with respect to the number of relays, for a packet error probability of $p=0.3$

by the proposed architecture. The first is a centralized approach that employs the cloud resources for the storage of keys, but must rely on the cloud provider to refrain from decoding the encrypted data stored in the cloud. In the proposed framework, this vulnerability risk is not present, since even though the keys will be handled by the cloud controller, the data will remain on the local data plane, within the relay cloud. The second approach proposes the decentralized distribution of keys among the users, but requires an arbitration entity for the key recovery. In the proposed framework, the cloud controller can play the role of the arbitrator, facilitating key management operations such as key recovery and revocation.

\section{Conclusions}

In this article, we have presented a general framework for the design of efficient cloud-assisted protocols in AAL environments. The proposed framework defines two planes of operation for control and data, thus delegating network coordination tasks to a central entity located at the cloud, while all data related operations take place locally at the nodes.

Within this framework, we have developed a novel MAC protocol that manages to fully exploit the potential of RLNC in a cooperative relay network. The obtained results have shown significant performance gains that become more prominent under challenging scenarios, when the channel conditions are harsh and only a few relays are available. Finally, we have also indicated other possible ways to exploit the potential gains offered by the cloudassisted network coordination, opening the road for many new applications.

\section{ACKNOWLEDGEMENTS}

This work has been funded by the research projects WSN4QoL (286047), SGR(2014 SGR 1551) and GEOCOM (TEC2011-27723-C02-01).

\section{REFERENCES}

[1] United Nations, Dept. of Economic and Social Affairs, Population Division, "World Population Prospects: The 2012 Revision, Volume I: Comprehensive Tables," ST/ESA/SER.A/336, 2013.

[2] P. Rashidi and A. Mihailidis, "A Survey on Ambient-Assisted Living Tools for Older Adults," IEEE Journal of Biomedical and Health Informatics, vol. 17, pp. 579-590, May 2013.

[3] G. Van Den Broek, F. Cavallo, and C. Wehrmann, AALIANCE Ambient Assisted Living Roadmap. Amsterdam, The Netherlands: IOS Press, Jan. 2010.

[4] M. Alam, M. B. I. Reaz, and M. A. M. Ali, "A Review of Smart Homes - Past, Present, and Future," IEEE Transactions on Systems, Man, and Cybernetics, Part C: Applications and Reviews, vol. 42, pp. 1190-1203, Nov. 2012.

[5] H. Pung, T. Gu, W. Xue, P. Palmes, J. Zhu, W. L. Ng, C. W. Tang, and N. H. Chung, "Context-aware middleware for pervasive elderly homecare," IEEE Journal on Selected Areas in Communications, vol. 27, pp. 510-524, May 2009.

[6] A. Wood, J. Stankovic, G. Virone, L. Selavo, Z. He, Q. Cao, T. Doan, Y. Wu, L. Fang, and R. Stoleru, "Context-aware wireless sensor networks for assisted living and residential monitoring," IEEE Network, vol. 22, pp. 26-33, July 2008. 
[7] M. Mulvenna, W. Carswell, P. McCullagh, J. Augusto, H. Zheng, P. Jeffers, H. Wang, and S. Martin, "Visualization of data for ambient assisted living services," IEEE Communications Magazine, vol. 49, pp. 110-117, Jan. 2011.

[8] C. Bachmann, M. Ashouei, V. Pop, M. Vidojkovic, H. Groot, and B. Gyselinckx, "Low-power wireless sensor nodes for ubiquitous long-term biomedical signal monitoring," IEEE Communications Magazine, vol. 50, pp. 20-27, Jan. 2012.

[9] R. Bassoli, H. Marques, J. Rodriguez, K. Shum, and R. Tafazolli, "Network coding theory: A survey," Communications Surveys Tutorials, IEEE, vol. 15, pp. 1950-1978, Fourth Quarter 2013.

[10] E. AbuKhousa, N. Mohamed, and J. Al-Jaroodi, "e-Health Cloud: Opportunities and Challenges," Future Internet, vol. 4, no. 3, pp. 621-645, 2012.

[11] J. Wan, C. Zou, S. Ullah, C.-F. Lai, M. Zhou, and X. Wang, "Cloud-enabled wireless body area networks for pervasive healthcare," IEEE Network, vol. 27, pp. 56-61, Sept. 2013.

[12] J. H. Lim, A. Zhan, J. Ko, A. Terzis, S. Szanton, and L. Gitlin, "A closed-loop approach for improving the wellness of lowincome elders at home using game consoles," IEEE Communications Magazine, vol. 50, pp. 44-51, Jan. 2012.

[13] A. Benharref and M. Serhani, "Novel Cloud and SOA-Based Framework for E-Health Monitoring Using Wireless Biosensors," IEEE Journal of Biomedical and Health Informatics, vol. 18, pp. 46-55, Jan. 2014.

[14] P. Dely, J. Vestin, A. Kassler, N. Bayer, H. Einsiedler, and C. Peylo, "CloudMAC - An OpenFlow based architecture for 802.11 MAC layer processing in the cloud," in Proc. of IEEE Globecom Workshops (GC Wkshps 2012), pp. 186-191, 2012.

[15] T. Uricar, T. Hynek, P. Prochazka, and J. Sykora, "Wirelessaware Network Coding: Solving a Puzzle in Acyclic Multistage Cloud Networks," in Proc. of the Tenth International Symposium on Wireless Communication Systems (ISWCS 2013), pp. 1-5, Aug. 2013. 